Journal of Statistical Physics, Vol. 11, No. 5, 1974

\title{
Spectral Representations for the Memory Kernel
} Characterizing Self-Diffusion

\author{
R. D. Mosteller ${ }^{1}$ and J. J. Duderstadt ${ }^{2}$
}

\author{
Received May 13, 1974
}

\begin{abstract}
Approximate spectral representations are developed for the memory kernel which characterizes self-diffusion. These spectral representations are based upon approximate eigenfunctions constructed via the Rayleigh variational principle. A heuristic model is developed first in an effort to provide physical insight into the nature of the approximations employed, and then a number of specific trial functions are examined. These trial functions include sums of identical one- and two-particle functions as well as linear combinations of hydrodynamical variables. The results from these spectral representations indicate that the long-time behavior of the memory kernel (and thereby of the momentum autocorrelation function) is sensitive to the long-range effects of the interparticle potential. In addition, the equivalence of most of these spectral representations to specific low-order perturbation approximations is demonstrated.
\end{abstract}

KEY WORDS: Brownian motion; Markovian approximations; memory kernel equations; momentum autocorrelation function; projection operator formalisms; self-diffusion.

This work was supported in part by the National Science Foundation under Grant GK-19360X.

${ }^{1}$ Department of Nuclear Science and Engineering, The Catholic University of America, Washington, D.C.

${ }^{2}$ Department of Nuclear Engineering, The University of Michigan, Ann Arbor, Michigan. 


\section{INTRODUCTION}

The momentum autocorrelation function

$$
\Phi(t) \equiv(\beta / 3 m)\left\langle\mathbf{p}_{\mathbf{1}} \cdot \mathbf{p}_{1}(t)\right\rangle, \quad \beta \equiv 1 / k_{B} T
$$

describes the motion of a test particle with mass $m$ and initial momentum $\mathbf{p}_{1}$ in a many-body system. Its time evolution can be described exactly by a memory kernel equation, ${ }^{(1)}$

$$
\dot{\Phi}(t)+\int_{0}^{t} d \tau K(t-\tau) \Phi(\tau)=0
$$

The memory kernel $K(t)$ is given formally as

$$
K(t) \equiv(\beta / 3 m)\left\langle\mathbf{F}_{1} \cdot\left\{\exp \left[i t\left(1-\mathscr{P}_{M}\right) L\right]\right\} \mathbf{F}_{1}\right\rangle
$$

where $\mathbf{F}_{1}$ is the initial force on the test particle and $L$ is the well-known Liouville operator, $i L_{\circ} \equiv\{H, \circ\}_{P B}$. The projection operator $\mathscr{P}_{M}$ was introduced by Zwanzig ${ }^{(1,3)}$ and Mori $^{(4)}$ and is defined by its action upon a general dynamical variable $\mathbf{G}$ as

$$
\mathscr{P}_{M} \mathbf{G} \equiv(\beta / 3 m) \mathbf{p}_{1}\left\langle\mathbf{p}_{1} \cdot \mathbf{G}\right\rangle
$$

No exact solution to the memory kernel equation (2) is possible, since it is equivalent to the coupled equations of motion for the entire $N$-body system $\left(N \sim 10^{23}\right)$. Consequently, approximations must be introduced for the memory kernel-for instance, perturbation expansions of the modified time evolution operator $\left\{\exp \left[i t\left(1-\mathscr{P}_{M}\right) L\right]\right\}$.

An alternate kind of approximation for the modified propagator $\exp \left\{i\left[\left(1-\mathscr{P}_{M}\right) L\right]\right\}$ consists in replacing it by an approximate spectral representation. Such an approach would appear to be particularly well suited for an investigation of long-time behavior, since all the long-time dynamics would be contained in the single term corresponding to the dominant eigenvalue.

If a Laplace transform in time is introduced,

$$
\tilde{\Phi}(s) \equiv \int_{0}^{\infty} d t e^{-s t} \Phi(t)
$$

then in the $s$ domain the memory kernel equation (2) takes the form

$$
\tilde{\Phi}(s)=1 /[s+\tilde{K}(s)]
$$

where $\widetilde{K}(s)$ is the transform of the memory kernel,

$$
\widetilde{K}(s)=\frac{\beta}{3 m}\left\langle\mathbf{F}_{1} \cdot \frac{1}{s-i\left(1-\mathscr{P}_{M}\right) L} \mathbf{F}_{1}\right\rangle
$$


If the complete sets of eigenvalues $\left\{\mu_{j}\right\}$ and eigenfunctions $\left\{\chi_{j}\right\}$ of the modified operator $i\left[\left(1-\mathscr{P}_{M}\right) L\right]$ are known, then the memory kernel can be calculated as

$$
\widetilde{K}(s)=\frac{\beta}{3 m} \sum_{j}\left\langle\mathbf{F}_{1} \cdot x_{j}\right\rangle \frac{1}{s+\mu_{j}}\left\langle\chi_{j} \cdot \mathbf{F}_{1}\right\rangle
$$

In the limit of long times this expression yields

$$
K(t) \underset{t \rightarrow \infty}{\longrightarrow}(\beta / 3 m)\left|\left\langle\mathbf{F}_{1} \cdot \boldsymbol{\chi}_{0}\right\rangle\right|^{2} e^{-\mu_{0} t}
$$

where $\mu_{0}$ is the least damped of the eigenvalues. All the time eigenvalues must lie in the left half of the complex $s$ plane for stable systems.

Normally, neither the eigenvalues nor eigenfunctions can be determined exactly. Often, for example, an infinite set of true eignfunctions is approximated by a finite set of approximate eigenfunctions. There are two wellknown methods for generating approximate eigenfunctions. One of these utilizes perturbation theory, while the other employs variational principles to construct approximate eigenfunctions from a given set of trial functions. However, in an effort to provide physical insight into the nature of such approximations, a heuristic development of an approximate spectral representation of $K(t)$ will be examined first.

\section{A HEURISTIC METHOD FOR GENERATING APPROXIMATE EIGENFUNCTIONS}

It is straightforward to demonstrate that the memory kernel $\widetilde{K}(s)$ can be rewritten as

$$
\begin{aligned}
\widetilde{K}(s)= & -\frac{1}{3 m} \int \frac{d^{3} k}{(2 \pi)^{3}} k^{2} \tilde{V}(k) \\
& \times \sum_{\alpha=2}^{N}\left\langle\left[\exp \left(i \mathbf{k} \cdot \mathbf{x}_{1}\right)\right] \frac{1}{s-i\left(1-\mathscr{P}_{M}\right) L} \exp \left(-i \mathbf{k} \cdot \mathbf{x}_{\alpha}\right)\right\rangle
\end{aligned}
$$

where $\tilde{V}(k)$ is the Fourier transform of the interparticle potential. The $\mathbf{x}_{1}$ dependence of the modified propagator $\left[s-i\left(1-\mathscr{P}_{M}\right) L\right]^{-1}$, of course, has been transformed into $\mathbf{k}$ dependence in Eq. (10). Since the ensemble average can be taken before the $\mathbf{k}$-space integration is performed, it is advantageous to find a function $\tilde{z}\left(\mathbf{k}, \mathbf{p}_{1}, \Gamma_{B}, s\right)$ such that

$$
\left[s-i\left(1-\mathscr{P}_{M}\right) L\right] \tilde{z}\left(\mathbf{k}, \mathbf{p}_{1}, \Gamma_{B}, s\right)=\exp \left(-i \mathbf{k} \cdot \mathbf{x}_{\alpha}\right)
$$

where $\Gamma_{B}$ is the ensemble variable for the $(N-1)$-particle bath; i.e., $\Gamma_{B} \equiv$ $\left[\mathbf{x}_{2}, \mathbf{x}_{3}, \ldots, \mathbf{x}_{N}, \mathbf{p}_{2}, \mathbf{p}_{3}, \ldots, \mathbf{p}_{N}\right]$. The inverse Fourier transform of Eq. (11),

$$
\left[s-i\left(1-\mathscr{P}_{M}\right) L\right] \tilde{z}\left(\mathbf{x}_{1}, \mathbf{p}_{1}, \Gamma_{B}, s\right)=\delta\left(\mathbf{x}_{1}-\mathbf{x}_{\alpha}\right)
$$


suggests that $\tilde{z}$ corresponds closely to a Green's function. It is apparent that at $t=0(s \rightarrow \infty)$

$$
z\left(\mathbf{x}_{1}, \mathbf{p}_{1}, \Gamma_{B}\right)=\delta\left(\mathbf{x}_{1}-\mathbf{x}_{\alpha}\right)
$$

But $\delta\left(\mathbf{x}_{1}-\mathbf{x}_{\alpha}\right)$ is just the microscopic probability density for the $\alpha$ th particle in $\mathbf{x}_{1}$ space, and the time correlation of this function is the well-known single-particle distribution function $G_{s}{ }^{(5,6)}$

Equation (13) suggests that the behavior of $z$ and of $G_{s}$ may be intimately related. For long times $G_{s}$ obeys a diffusion equation,

$$
(\partial / \partial t) \widetilde{G}_{s}\left(\mathbf{k}, \mathbf{x}_{\alpha}, t\right)=-k^{2} D_{s} \widetilde{G}_{s}\left(\mathbf{k}, \mathbf{x}_{\alpha}, t\right)
$$

where $D_{s}$ is the coefficient of self-diffusion. These results suggest that the least-damped eigenvalue of the modified propagator is approximately

$$
\mu_{0} \cong k^{2} D_{s}
$$

with a corresponding approximate eigenfunction

$$
\chi_{0} \cong \exp \left(-i \mathbf{k} \cdot \mathbf{x}_{\alpha}\right)
$$

These approximations, in conjunction with Eqs. (9) and (10), imply that

$$
K(t) \underset{t \rightarrow \infty}{\longrightarrow}-\frac{n}{3 m} \int \frac{d^{3} k}{(2 \pi)^{3}} k^{2} \tilde{V}(k) \tilde{g}(k) \exp \left(-k^{2} D_{s} t\right)
$$

where $n$ is the average density of particles in the system, and $\tilde{g}(k)$ is the static pair correlation function,

$$
n \tilde{g}(k) \equiv \sum_{\alpha=2}^{N}\left\langle\exp \left[i \mathbf{k} \cdot\left(\mathbf{x}_{1}-\mathbf{x}_{\alpha}\right)\right]\right\rangle
$$

This long-time behavior of $K(t)$ can be evaluated quite easily, since the exponential damping restricts the dominant contribution to the integral to the region near the origin in $\mathbf{k}$ space. This result, in turn, implies that the long-time behavior of the memory kernel (and thereby of the momentum autocorrelation function) is determined largely by the long-range effect of the interparticle potential.

Apart from its heuristic derivation, the most severe limitation upon Eq. (17) is that it describes only those systems whose interparticle potentials possess Fourier transforms. At the same time, it does contain one surprising implication-it suggests that the asymptotic behavior of the memory kernel (and thereby of the momentum autocorrelation function) is different in systems characterized by different interparticle potentials.

In many cases, the asymptotic form of the momentum autocorrelation function can be inferred directly from the long-time behavior of the memory 
kernel $^{(7)}$ (see also the appendix). If $\Phi(t)$ decays according to a power law, the asymptotic tails of $\Phi(t)$ and $K(t)$ are of opposite sign. Furthermore, $\tilde{g}(k)$ approaches a negative constant for small $k$,

$$
\tilde{g}(k) \underset{k \rightarrow 0}{\longrightarrow}\left(\frac{\chi_{T}}{\beta}-\frac{1}{n}\right)<0
$$

where $\chi_{T}$ is the isothermal compressibility of the system. Equation (17) therefore implies that the asymptotic tail of the momentum autocorrelation function will be strictly negative in a system characterized by a long-range repulsive potential.

For the sake of illustration, consider two particular interparticle potentials. If $\mathbf{Y}(r)$ is a finite step,

$$
V(r)= \begin{cases}\mathbf{V}_{0}, & 0 \leq r \leq r_{0} \\ 0, & r>r_{0}\end{cases}
$$

then the memory kernel has the asymptotic form

$$
K(t)_{t \rightarrow \infty}^{\longrightarrow} \frac{V_{0} r_{0}^{3}}{12 m \sqrt{\pi}}\left(1-\frac{n \chi_{T}}{\beta}\right)\left(D_{s} t\right)^{-5 / 2}
$$

and hence, from the appendix,

$$
\Phi(t)_{t \rightarrow \infty}-\frac{\beta V_{0} r_{0}^{3} m}{12\left(\pi D_{s}\right)^{1 / 2}}\left(\beta-n \chi_{T}\right) t^{-5 / 2}
$$

If it is a Coulomb potential,

$$
V(r)=Q_{0} / r=\lim _{\varepsilon \rightarrow 0}\left(Q_{0} / r\right) e^{-\varepsilon r}
$$

then

$$
K(t)_{t \rightarrow \infty} \frac{Q_{0} \sqrt{\pi}}{6 m}\left(1-\frac{n_{\chi_{T}}}{\beta}\right)\left(D_{s} t\right)^{-3 / 2}
$$

and therefore

$$
\Phi(t)_{t \rightarrow \infty} \rightarrow-\frac{\beta Q_{0} m}{6}\left(\beta-n \chi_{T}\right)\left(D_{s} / \pi\right)^{1 / 2} t^{-3 / 2}
$$

Consequently, the momentum autocorrelation function for a system characterized by a Coulomb potential decays according to the same power law as it does for a system of hard spheres. ${ }^{(8-10)}$ Unfortunately, the predictions of Eq. (17) cannot be compared directly with this last result since a hard-sphere potential does not possess a Fourier transform. 


\section{METHODS FOR GENERATING APPROXIMATE EIGENFUNCTIONS}

Perhaps the most common method for generating approximate eigenfunctions is that based upon perturbation theory. In this approach, the approximate eigenfunctions are expressed as linear combinations of the known eigenfunctions of a related operator. In the spectral representation for the memory kernel, Eq. (8), the eigenfunctions of the modified propagator appear only within the ensemble averages. Consequently, since the eigenfunctions of the related operator form a complete set and are (by assumption) already known, a more efficient approach would be to express $\tilde{K}(s)$ in terms of these known eigenfunctions and then perform a perturbation expansion on the modified propagator $\left[s-i\left(1-\mathscr{P}_{M}\right) L\right]^{-1}$. This approach was investigated in a previous paper. ${ }^{(11)}$ It leads to meaningful long-time results for some specific approximations but not for others.

Another method for generating approximate eigenfunctions and eigenvalues is based upon the Rayleigh variational principle,

$$
\lambda=J\left[\Psi^{\prime}\right]=\left\langle\Psi^{\dagger} \cdot \hat{\mathcal{O}} \Psi\right\rangle\left\langle\left\langle\Psi^{\dagger} \cdot \Psi\right\rangle\right.
$$

If the operator $\hat{\mathcal{O}}$ is self-adjoint, a first-order error in the trial function $\Psi$ will produce only a second-order error in the approximate eigenvalue $\lambda$. In addition, the solutions of the eigenvalue problem will correspond to extrema of the functional $J$. If the trial functions $\Psi$ are restricted to functions orthogonal to $\mathbf{p}_{1}$, then this same scheme can be applied to the modified operator $\left[\left(1-\mathscr{P}_{M}\right) L\right]$ since it is self-adjoint in that space.

Zwanzig ${ }^{(12)}$ was the first to apply variational principles to the construction of eigenfunctions for the Liouville operator $L$. He chose his trial functions as sums of real, identical one-particle functions,

$$
\Psi=\Psi^{\rho}=\sum_{\alpha=1}^{N} \psi\left(\mathbf{x}_{\alpha}, \mathbf{p}_{\alpha}\right) \equiv \sum_{\alpha=1}^{N} \psi(\alpha)
$$

When this trial function is inserted into the functional for the modified operator $\left[\left(1-\mathscr{P}_{M}\right) L\right]$ and its first variation $\delta J / \delta \psi^{\dagger}$ is set to zero, there results

$$
\begin{aligned}
\frac{i}{m} \mathbf{p}_{1} & \cdot \frac{\partial}{\partial \mathbf{x}_{1}} \psi(1)-i\left\langle\mathbf{F}_{1}\right\rangle \cdot \frac{\partial}{\partial \mathbf{p}_{1}} \psi(1)-\frac{i}{m} \mathbf{p}_{1} \cdot\left\langle\frac{\partial}{\partial \mathbf{x}_{1}} \psi(1)\right\rangle \\
& =\lambda\left\{\psi(1)+(N-1) \int d(2) \frac{f_{2}(1,2)}{f_{1}(1)} \psi(2)\right\}
\end{aligned}
$$

where $f_{j}$ is the $j$-particle distribution function in phase space,

$$
f_{j}(1,2, \ldots, j) \equiv \int d(j+1) \cdots \int d(N) f_{0}(1,2, \ldots, N)
$$


and $f_{0}$ is the equilibrium phase-space distribution function for the system.

The projection operator $\mathscr{P}_{M}$ contributes only to the term $\left\langle\partial \psi(1) / \partial \mathbf{x}_{1}\right\rangle$ in Eq. (28). In a homogeneous system, however, this term is zero. For the trial function (27), therefore, the functional (26) gives approximate eigenvalues and eigenfunctions which are just the approximate eigenvalues and eigenfunctions of the Liouville operator itself. Such results are equivalent to a first-order perturbation approximation in which the memory kernel $K(t)$ is approximated by the force autocorrelation function $k(t)$,

$$
k(t) \equiv(\beta / 3 m)\left\langle\mathbf{F}_{1} \cdot \mathrm{e}^{i t L} \mathbf{F}_{1}\right\rangle
$$

Interestingly enough, the eigenfunetions of the Liouville operator can lead to an expression for the memory kernel which is very similar to that obtained by heuristic means. Consider a one-particle eigenfunction of the form

$$
\psi(\alpha)=\left[\exp \left(i \mathbf{k} \cdot \mathbf{x}_{\alpha}\right)\right] \rho\left(\mathbf{p}_{\alpha}\right)
$$

When this expression is substituted into Eq. (28), there results

$$
\frac{i}{m} \mathbf{k} \cdot \mathbf{p}_{\alpha} \rho\left(\mathbf{p}_{\alpha}\right)=\lambda \rho\left(\mathbf{p}_{\alpha}\right)+\lambda n \tilde{g}(k) \int d^{3} p_{\alpha}{ }^{\prime} M\left(\mathbf{p}_{\alpha}{ }^{\prime}\right) \rho\left(\mathbf{p}_{\alpha}{ }^{\prime}\right)
$$

where $M(\mathbf{p})$ is the Maxwellian momentum distribution function. If a new function $\xi\left(\mathbf{p}_{\alpha}\right)$ is defined as ${ }^{(12)}$

$$
\xi\left(\mathbf{p}_{\alpha}\right) \equiv M\left(\mathbf{p}_{\alpha}\right) \rho\left(\mathbf{p}_{\alpha}\right)+n \tilde{g}(k) M\left(\mathbf{p}_{\alpha}\right) \int d^{3} p_{\alpha}{ }^{\prime} M\left(\mathbf{p}_{\alpha}{ }^{\prime}\right) \rho\left(\mathbf{p}_{\alpha}{ }^{\prime}\right)
$$

then Eq. (32) assumes the form

$$
\frac{i}{m} \mathbf{k} \cdot \mathbf{p}_{\alpha} \xi\left(\mathbf{p}_{\alpha}\right)-i \nu_{p}{ }^{2} \mathbf{k} \cdot\left[\frac{\partial}{\partial \mathbf{p}_{\alpha}} M\left(\mathbf{p}_{\alpha}\right)\right] \int d^{3} p_{\alpha}{ }^{\prime} \xi\left(\mathbf{p}_{\alpha}{ }^{\prime}\right)=\lambda \xi\left(\mathbf{p}_{\alpha}\right)
$$

where

$$
\nu_{p}^{2} \equiv-\frac{1}{m \beta} \frac{n \tilde{g}(k)}{1+n \tilde{g}(k)}
$$

Equation (34) has exactly the same structure as the linearized Vlasov equation.

For the particular case of a plasma,

$$
\nu_{p}^{2} \rightarrow \omega_{p}^{2} / k^{2}
$$

where $\omega_{p}$ is the plasma frequency. For long times $(s \rightarrow 0)$, the dispersion law for a plasma is proportional to $\left\{s^{2}+2 s \gamma_{\mathrm{L}}(k)+\left[\omega_{p}{ }^{2}+(3 / m \beta) k^{2}\right]\right\}$, where $\gamma_{\mathrm{L}}$ is the Landau damping, ${ }^{(13)}$

$$
\gamma_{\mathbf{L}}(k)=(2 \pi)^{1 / 2} \omega_{p}\left(\frac{m \beta \omega_{p}^{2}}{2 k^{2}}\right) \exp \left(-\frac{3}{2}-\frac{m \beta \omega_{p}^{2}}{2 k^{2}}\right)
$$


For long times, however, the oscillatory contributions to this dispersion law average to zero, and the memory kernel assumes the asymptotic form

$$
K(t) \underset{i \rightarrow \infty}{\longrightarrow}-\frac{n}{3 m} \int \frac{d^{3} k}{(2 \pi)^{3}} k^{2} V(k) \tilde{g}(k) e^{-\gamma_{L}(k) t}
$$

This result is very similar in form to that obtained from heuristic arguments, Eq. (17).

The projection operator $\mathscr{P}_{M}$ has not been involved in the calculation of these approximate eigenfunctions. In fact, this last approach is strictly equivalent to the approximation

$$
K(t) \underset{t \rightarrow \infty}{\longrightarrow} k(t)
$$

The term $\left\langle\partial \psi / \partial \mathbf{x}_{1}\right\rangle$ disappears from Eq. (28) because $\psi$ depends upon the phase-space coordinates of only a single particle. A refinement of this approach is to choose the trial function as a sum of identical two-particle functions,

$$
\Psi(1,2, \ldots, N)=\sum_{\alpha=1}^{N} \sum_{\gamma=1 \neq \alpha}^{N} \psi(\alpha, \gamma)
$$

Even when it is assumed that $\psi(\alpha, \gamma)$ is real and is symmetric under particle interchange, the resulting eigenvalue equation is quite complicated. In addition to its formal complexity, the equation involves three- and four-particle distribution functions. Consequently, no further development of this approach will be considered here.

Another method for generating approximate eigenfunctions involves restricting the admissible trial functions to linear combinations of mutually orthogonal hydrodynamic variables. ${ }^{(14)}$ Such a scheme is actually rather closely related to the formalism of Mori. ${ }^{(4)}$ Suppose one wishes to compute the time correlation function of a dynamical variable $a_{1}$ using approximate eigenvalues $\lambda$ and eigenfunctions $\psi_{\lambda}$

$$
\tilde{\Phi}_{a}(s)=\left\langle a_{1} \tilde{a}_{1}(s)\right\rangle\left\langle\left\langle a_{1}{ }^{2}\right\rangle=\sum_{\lambda}\left[\left\{\left|\left\langle a_{1} \mid \psi_{\lambda}\right\rangle\right|^{2} /\left\langle a_{1}{ }^{2}\right\rangle\right\} /(s-\lambda)\right]\right.
$$

Then by seeking trial functions which are linear combinations of mutually orthogonal variables $a_{1}, a_{2}, \ldots a_{I}$ (including $a_{1}$ itself)

$$
\psi=\sum_{i=1}^{I} c_{i} a_{i}
$$

and demanding $\left(\delta J / \delta c_{j}^{+}\right)=0$, one arrives at an eigenvalue condition

$$
\|\boldsymbol{\Omega}-\boldsymbol{\omega} \mathbf{I}\|=0
$$


where

$$
\boldsymbol{\Omega} \equiv\langle\mathbf{a} * \dot{a}\rangle \cdot\left\langle\mathbf{a}^{*} \mathbf{a}\right\rangle^{-1}, \quad \mathbf{a}=\operatorname{col}\left[a_{1}, \ldots, a_{1}\right]
$$

Hence one finds that such trial functions lead to an expression for $\tilde{\Phi}_{a}(s)$ of the form

$$
\tilde{\Phi}_{a}(s)=\left\langle a_{1} \hat{e}_{1} \cdot[s-i \Omega]^{-1} \cdot a_{1} \hat{e}_{1}\right\rangle\left\langle\left\langle a_{1}{ }^{2}\right\rangle, \quad \hat{e}_{1}=\operatorname{col}[1,0,0, \ldots, 0]\right.
$$

which is identical to Mori's description when the damping term is neglected.

The eigenvalues $\omega_{j}$ are purely imaginary, and it is straightforward to demonstrate that the resulting expression for $\Phi_{\mathrm{a}}(t)$ is simply an oscillatory function. Such behavior is valid only for very short times. The variational approach utilizing hydrodynamic variables therefore corresponds to a shorttime approximation and, in fact, is equivalent to approximating the damping kernel as zero in the generalized Langevin equation for the trial function. This last conclusion also has been reached by Bost in his detailed study of the frequency and damping matrices. ${ }^{(15)}$

This same class of trial functions can also be used to generate approximate eigenfunctions of the modified propagator $\left(1-\mathscr{P}_{M}\right) L$, provided the variable $a_{1}$ is eliminated from the expansion set:

$$
\Psi=\sum_{i=2}^{I} c_{i} a_{i}
$$

The variational principle then yields an eigenvalue condition

$$
\left\|\boldsymbol{\Omega}_{2}-\omega \mathbf{l}\right\|=0
$$

where $\boldsymbol{\Omega}_{2}$ is defined as before with $\mathbf{a} \rightarrow \mathbf{a}^{\prime}=\operatorname{col}\left[a_{2}, \ldots, a_{N}\right]$. If these eigenfunctions and eigenvalues are used to calculate $\widetilde{K}(s)$, then it is evident that this corresponds to

$$
\tilde{\Phi}_{a}(s)=\left[s+\left\langle\dot{a}_{1} \hat{e}_{1} \cdot\left(s-i \boldsymbol{\Omega}_{2}\right)^{-1} \dot{a}_{1} \hat{e}_{1}\right\rangle /\left\langle a_{1}{ }^{2}\right\rangle\right]^{-1}
$$

which is closely related to Mori's continued fraction representation ${ }^{(16)}$ and corresponds once again to a short-time estimate.

Since the use of such hydrodynamic trial functions yields descriptions which have already been investigated within the framework of the Mori formalism, we will not pursue more explicit investigations of their application to the momentum autocorrelation function.

\section{SUMMARY}

The results of this paper fall into two categories-the heuristic model of Section 2 and the formal attempts to generate eigenfunctions in Section 3. These latter attempts appear to be, at best, equivalent to more straightforward perturbation approximations. As might be expected, spectral representations of the memory kernel involving approximate eigenfunctions 
generated by perturbation expansions are equivalent to more direct perturbation approximations for the modified propagator $\left[s-i\left(1-\mathscr{P}_{M}\right) L\right]^{-1}$. In addition, spectral representations based on eigenfunctions generated from a Rayleigh variational principle are equivalent to low-order perturbation approximations for many different trial functions. Specifically, trial functions composed of sums of one-particle functions or of hydrodynamic variables which evolve in a space orthogonal to the initial momentum of the test particle approximate the eigenfunctions of the modified operator $\left[\left(1-\mathscr{P}_{M}\right) L\right]$ by those of the Liouville operator. $L$. Such approximations are strictly equivalent to a first-order perturbation approximation in which the memory kernel $K(t)$ is approximated by the force autocorrelation function $k(t)$. In addition, it was noted that the method employed by Nossal and Zwanzig to generate eigenfunctions for the Liouville operator from trial functions composed of hydrodynamic variables is equivalent to approximating the damping matrix as zero in Mori's generalized Langevin equation for the trial function.

The heuristic model, on the other hand, offers a simple prescription for calculating the memory kernel in any system characterized by a Fouriertransformable interparticle potential. In many instances, once the particular long-time form of the memory kernel is known, the corresponding asymptotic behavior of the momentum autocorrelation function can be found directly from the results given in the appendix. The most surprising result from the heuristic model is its suggestion that the memory kernel (and thereby the momentum autocorrelation function) exhibits different asymptotic behavior in systems characterized by different interparticle potentials.

The heuristic model, in conjunction with Eq. (38) and the results from previous work, ${ }^{(11)}$ suggests that in many systems the memory kernel has the asymptotic form

$$
K(t) \underset{t \rightarrow \infty}{\longrightarrow}-\frac{n}{3 m} \int \frac{d^{3} k}{(2 \pi)^{3}} k^{2} \tilde{V}(k) \tilde{g}(k) \exp \left[-k^{2}\left(\nu+D_{s}\right) t\right]
$$

where $\nu$ and $D_{s}$ are the kinematic viscosity and the coefficient of self-diffusion, respectively. A similar conclusion about the momentum autocorrelation function itself,

$$
\Phi(t) \underset{t \rightarrow \infty}{\longrightarrow} c\left[\left(\nu+D_{s}\right) t\right]^{-3 / 2}
$$

has been reached in a number of other investigations. ${ }^{(8,9,17-21)}$

\section{APPENDIX A. AN ASYMPTOTIC RELATIONSHIP BETWEEN $\Phi(t)$ and $K(t)$}

Corngold has derived a relationship between the long-time behavior of the memory kernel and that of the momentum autocorrelation function for the case in which $\Phi(t)$ decays asymptotically according to a power law. ${ }^{(7)}$ The 
behavior of the two functions is similar, since in that case it is determined by a branch cut along the negative real axis in $s$ space. Specifically, he demonstrated that if

$$
\Phi(t) \underset{t \rightarrow \infty}{\longrightarrow} c_{0} t^{-\gamma}, \quad \gamma>0
$$

then

$$
K(t) \underset{t \rightarrow \infty}{\longrightarrow} \begin{cases}-\left[\frac{1-\gamma}{\pi c_{0}^{2}} \sin (\gamma \pi)\right] \frac{1}{t^{2}} \Phi(t), & 0<\gamma<1 \\ -\left(\frac{1}{m \beta D_{s}}\right)^{2} \Phi(t), & \gamma \geqslant 1\end{cases}
$$

This result implies that the asymptotic tails of $K(t)$ and $\Phi(t)$ are of opposite sign, independent of $c_{0}$ and $\gamma$.

An alternative but completely equivalent expression for the memory kernel is

$$
K(t) \underset{t \rightarrow \infty}{\longrightarrow} \begin{cases}-\left[\frac{1-\gamma}{\pi c_{0}{ }^{2}} \sin (\gamma \pi)\right] \frac{1}{t^{2}} \Phi(t), & 0<\gamma<1 \\ -\left(\frac{1}{c_{0} \ln t}\right)^{2} \Phi(t), & \gamma=1 \\ -\left(\frac{1}{m \beta D_{s}}\right)^{2} \Phi(t), & \gamma>1\end{cases}
$$

since the coefficient of self-diffusion,

$$
D_{s}=(1 / m \beta) \int_{0}^{\infty} d t \Phi(t)
$$

contains a logarithmic divergence when $\gamma=1$. In many cases, if the longtime form of the memory kernel is known, the asymptotic behavior of the momentum autocorrelation function can be inferred directly from Eqs. (A.1) and (A.3).

An additional property of the memory kernel can be established for the cases $\gamma \leqslant 1$. From Eq. (A.1) it is obvious that

Therefore, from Eq. (6),

$$
\tilde{\Phi}(s) \underset{s \rightarrow 0}{\longrightarrow} \infty
$$

$$
\int_{0}^{\infty} d t K(t)=\widetilde{K}(s)=0
$$

\section{ACKNOWLEDGMENTS}

The authors gratefully acknowledge many fruitful discussions with A. Z. Akcasu and C. M. Bost, Jr. In addition, one of the authors (R.D.M.) 
wishes to acknowledge the financial support provided by an AEC-NSE fellowship during a period in which much of this research was carried on.

\section{REFERENCES}

1. R. Zwanzig, in Lectures in Theoretical Physics, Vol. III, W. E. Brittin, W. B. Downs, and J. Downs (eds.), Interscience, New York (1961).

2. R. Zwanzig, J. Chem. Phys. 33:1338 (1960).

3. R. Zwanzig, Phys, Rev. 124:983 (1961).

4. H. Mori, Progr. Theor. Phys. (Kyoto) 33:423 (1965).

5. G. E. Uhlenbeck and L. S. Ornstein, Phys. Rev. 36:823 (1930) and references contained therein.

6. S. Chandrasekhar, Rev. Mod. Phys. 15:1 (1943) and references contained therein.

7. N. Corngold, Phys. Rev. A 6:1570 (1972).

8. B. J. Alder and T. E. Wainwright, Phys. Rev. Lett. 18:988 (1967).

9. B. J. Alder and T. E. Wainwright, Phys. Rev. A 1:18 (1970).

10. B. J. Alder, D. M. Gass, and T. E. Wainwright, J. Chem, Phys. 53:3813 (1970).

11. R. D. Mosteller and J. J. Duderstadt, J. Stat. Phys. 9:197 (1973).

12. R. Zwanzig, Phys. Rev. 144:170 (1966).

13. L. Landau, J. Phys. (USSR) 10:25 (1946).

14. R. Nossal and R. Zwanzig, Phys. Rev. 157:120 (1967).

15. C. M. Bost, Jr., Ph.D. Thesis, University of Michigan (1973).

16. H. Mori, Progr. Theor. Phys. (Kyoto) 34:399 (1965).

17. B. J. Alder and T. E. Wainwright, J. Phys. Soc. Japan, Suppl. 26:267 (1968).

18. J. R. Dorfman and E. G. D. Cohen, Phys. Rev. Lett. 25:1257 (1970).

19. M. H. Ernst, E. H. Hauge, and J. M. J. van Leeuwen, Phys. Rev. Lett. 25:1254 (1970).

20. M. H. Ernst, E. H. Hauge, and J. M. J. van Leeuwen, Phys. Rev. A 4:2055 (1971).

21. G. F. Mazenko, Phys. Rev. A 7:209 (1973). 